\title{
OPEN Children's erythrocyte fatty acids are associated with the risk of islet autoimmunity
}

\author{
Sari Niinistö ${ }^{1 凶}$, Iris Erlund ${ }^{2}$, Hye-Seung Lee ${ }^{3}$, Ulla Uusitalo ${ }^{3}$, Irma Salminen ${ }^{2}$, \\ Carin Andrén Aronsson ${ }^{4}$, Hemang M. Parikh ${ }^{3}$, Xiang Liu ${ }^{3}$, Sandra Hummel ${ }^{5}$, Jorma Toppari ${ }^{6}$, \\ Jin-Xiong She ${ }^{3}$, Åke Lernmark ${ }^{4}$, Annette G. Ziegler ${ }^{5}$, Marian Rewers ${ }^{8}$, Beena Akolkar ${ }^{9}$, \\ Jeffrey P. Krischer ${ }^{3}$, David Galas ${ }^{10}$, Siba Das ${ }^{10}$, Nikita Sakhanenko ${ }^{10}$, Stephen S. Rich ${ }^{11}$, \\ William Hagopian ${ }^{10}$, Jill M. Norris ${ }^{12,31}$, Suvi M. Virtanen ${ }^{1,13,14,31}$ \& the TEDDY Study Group*
}

Our aim was to investigate the associations between erythrocyte fatty acids and the risk of islet autoimmunity in children. The Environmental Determinants of Diabetes in the Young Study (TEDDY) is a longitudinal cohort study of children at high genetic risk for type 1 diabetes $(n=8676)$ born between 2004 and 2010 in the U.S., Finland, Sweden, and Germany. A nested case-control design comprised 398 cases with islet autoimmunity and 1178 sero-negative controls matched for clinical site, family history, and gender. Fatty acids composition was measured in erythrocytes collected at the age of 3,6 , and 12 months and then annually up to 6 years of age. Conditional logistic regression models were adjusted for HLA risk genotype, ancestry, and weight z-score. Higher eicosapentaenoic and docosapentaenoic acid ( $n-3$ polyunsaturated fatty acids) levels during infancy and conjugated linoleic acid after infancy were associated with a lower risk of islet autoimmunity. Furthermore, higher levels of some even-chain saturated (SFA) and monounsaturated fatty acids (MUFA) were associated with increased risk. Fatty acid status in early life may signal the risk for islet autoimmunity, especially $\mathrm{n}-3$ fatty acids may be protective, while increased levels of some SFAs and MUFAs may precede islet autoimmunity.

$\begin{array}{ll}\text { Abbreviations } \\ \text { ALA } & \text { Alphalinolenic acid } \\ \text { CLA } & \text { Conjugated linoleic acid } \\ \text { DPA } & \text { Docosapentaenoic acid } \\ \text { DHA } & \text { Docosahexaenoic acid } \\ \text { DMA } & \text { Dimethylacetal } \\ \text { EPA } & \text { Eicosapentaenoic acid } \\ \text { GADA } & \text { Glutamic acid decarboxylase } \\ \text { HLA } & \text { Human leukocyte antigen } \\ \text { IAA } & \text { Insulin autoantibody }\end{array}$

${ }^{1}$ Health and Well-Being Promotion Unit, Public Health and Welfare Department, Finnish Institute for Health and Welfare, P.O. Box 30, 00271 Helsinki, Finland. ${ }^{2}$ Department of Government Services, Finnish Institute for Health and Welfare, Helsinki, Finland. ${ }^{3}$ Health Informatics Institute, Morsani College of Medicine, University of South Florida, Tampa, USA. ${ }^{4}$ Department of Clinical Sciences, Lund University, CRC, Skåne University Hospital, Malmö, Sweden. ${ }^{5}$ Institute of Diabetes Research, Helmholtz Zentrum München and Forschergruppe Diabetes, Klinikum Rechts Der Isar, Technische Universität München and Forschergruppe Diabetes e.V., Munich, Germany. ${ }^{6}$ Department of Physiology, University of Turku, Turku, Finland. ${ }^{8}$ Barbara Davis Center for Childhood Diabetes, University of Colorado School of Medicine, Aurora, USA. ${ }^{9}$ National Institute of Diabetes and Digestive and Kidney Diseases, National Institutes of Health, Bethesda, MD, USA. ${ }^{10}$ Pacific Northwest Research Institute, Seattle, WA, USA. ${ }^{11}$ University of Virginia School of Medicine, Virginia, USA. ${ }^{12}$ Department of Epidemiology, Colorado School of Public Health, University of Colorado Denver, Aurora, USA. ${ }^{13}$ Faculty of Social Sciences/Health Sciences, Tampere University and Center for Child Health Research, Tampere University and Tampere University Hospital, Tampere, Finland. ${ }^{14}$ The Science Center of Pirkanmaa Hospital District, Tampere, Finland. ${ }^{31}$ These authors jointly supervised this work: Jill M. Norris and Suvi M. Virtanen. *A list of authors and their affiliations appears at the end of the paper. ${ }^{\circledR}$ email: sari.niinisto@thl.fi 
$\begin{array}{ll}\text { IA-2A } & \text { Insulinoma-associated antigen-2 } \\ \text { LA } & \text { Linoleic acid } \\ \text { MUFA } & \text { Monounsaturated fatty acid } \\ \text { PUFA } & \text { Polyunsaturated fatty acid } \\ \text { SFA } & \text { Saturated fatty acid } \\ \text { SNP } & \text { Single nucleotide polymorphism } \\ \text { TEDDY } & \text { The Environmental Determinants of Diabetes in the Young Study }\end{array}$

Fatty acids are important constituents of complex lipids and cell membranes, affecting their physiological properties and cellular functions. They are also well-established precursors of lipid mediators, e.g. eicosanoids, which are involved in various inflammatory reactions and affect immunity, lipid and glucose metabolism, as well as insulin responses ${ }^{1-4}$. Fatty acids may play a role in the development of type 1 diabetes, an autoimmune disease characterized by the destruction of pancreatic insulin-producing beta cells. The strongest evidence concerns long-chain $n-3$ polyunsaturated fatty acid (PUFA) intake or status during infancy and childhood, which protected from islet autoimmunity ${ }^{5-7}$, and showed an interaction with breastfeeding ${ }^{7}$. However, $\mathrm{n}-3$ PUFAs were not associated with the progression from islet autoimmunity to type 1 diabetes ${ }^{8}$. In addition, other fatty acids than $n-3$ PUFAs have been associated with islet autoimmunity. Serum breast milk-derived fatty acids during infancy were protectively associated with primary insulin autoimmunity ${ }^{7}$, while dairy-derived fatty acids during later childhood were directly associated with islet autoimmunity ${ }^{9}$. Also, metabolomic studies investigating other lipids have reported that early metabolic lipid dysregulation preceding islet autoimmunity in children who later progressed to type 1 diabetes ${ }^{10-14}$.

Different types of fatty acids biomarkers have been used in previous studies, most commonly from serum or erythrocytes. Erythrocyte fatty acid composition is considered the most stable biomarker, reflecting longterm dietary intake or endogenous biosynthesis and metabolism several weeks or months back ${ }^{15}$. Some of the individual fatty acids are more useful as dietary biomarkers than others, because they reflect changes in dietary intake better. This includes fatty acids belonging to the $n-6$ and $n-3$ pathways ${ }^{16,17}$. In line with the definition, the essential fatty acids $n-6$ linoleic acid (LA) and $n-3$ alpha-linolenic acid (ALA) are solely obtained from the diet. These compounds are metabolized by the $n-3$ and $n-6$ pathways to form longer chain fatty acids ${ }^{18}$, which serve as precursors for lipid mediators. Main dietary sources of LA and ALA are vegetable oils, while longer chain $n-3$ fatty acids eicosapentaenoic (EPA), docosapentaenoic (DPA) and docosahexaenoic acid (DHA) are mainly obtained from marine foods. Other types of fatty acid biomarkers, e.g. the odd-chain fatty acids pentadecanoid acid (15:0) and heptadecanoid acid (17:0), as well as conjugated linoleic acid (CLA) (18:2n-7), have been applied as biomarkers of dairy fat ${ }^{19,20}$, although it is well known that fatty acids are rarely specific for any dietary source and they are often produced endogenously also $^{21-23}$. This is the case especially for the largest pool of fatty acids on cell membranes, the even-chain saturated fatty acids (SFA). The even-chain SFA and monounsaturated fatty acids (MUFA) are not considered good biomarkers of dietary intake, because they mostly reflect endogenous fatty acid metabolism and biosynthesis in the liver by a process that produces fatty acids from glucose mainly (de novo lipogenesis), as well as metabolism of shorter chain fatty acids ${ }^{24}$.

Type 1 diabetes may consist of different disease endotype ${ }^{25}$. First emerging autoantibody may reflect different etiology of endotypes, and different genes and environmental exposures may be associated with them ${ }^{7,26,27}$. The aim of the current study was to evaluate the associations of erythrocyte fatty acid composition during infancy and childhood with the different types of islet autoimmunity. Our hypothesis was that the long-chain $n-3$ PUFAs are associated with reduced risk.

\section{Results}

Erythrocyte fatty acid composition in infancy and the risk of the risk islet autoimmunity. Characteristics of children by matching factors are presented in Table 1, and erythrocyte fatty acid status in children in Supplementary information Table 1. Higher proportion of EPA and DPA at 3 months was associated with a lower risk for islet autoimmunity. In contrast, oleic acid $(18: 1 n-9)$ at 3 months and palmitic acid (16:0) at 6 months were associated with an increased risk of islet autoimmunity (Table 2). Erythrocyte fatty acid composition of infants differed according to breastfeeding status defined as consumption of any breastmilk (yes/ no) at the age of 3 or 6 months (Table 3). Non-breastfed infants exhibited higher levels of oleic acid $(18: 1 n-9)$ and palmitic acid (16:0) than breastfed children, and these fatty acids were associated with an increased risk of islet autoimmunity. ALA $(18: 3 n-3)$, LA $(18: 2 n-6)$ and docosanoid acid (22:0) showed an interaction with breastfeeding at 3 months on the risk of islet autoimmunity (ALA $p=0.024$, LA $p=0.038$, docosanoid acid $p=0.027$ ). In non-breastfed infants, ALA (OR 0.35, 95\% CI 0.15-0.83) and LA $(0.18,0.04-0.76$ ) were associated with a lower risk of islet autoimmunity, while no associations were observed in breastfed infants (ALA 1.09, 0.64-1.84; LA 1.06, 0.42-2.68). Docosanoid acid (22:0) was associated with an increased risk in non-breastfed infants (non-breastfed 3.31, 1.08-10.14; breastfed 0.82, 0.41-1.64).

Infants' fatty acid status at the age of 3 and 6 months was not associated with the risk of multiple islet autoimmunity (Supplementary information Table 2), but some associations with IAA first and GADA first outcomes were observed. DPA $(22: 5 n-3)$ at 3 months showed a protective association with IAA first autoimmunity, while a high ratio of $n-6: n-3$ PUFA at 6 months was associated with a higher risk (Supplementary information Table 3). For GADA first, a protective association was observed for AA $(20: 4 n-6)$ and adrenic acid $(22: 4 n-6)$ at 6 months, while myristic acid (14:0) at 6 months was associated with a higher risk (Supplementary information Table 4).

Erythrocyte fatty acid composition in children aged 1-6 years and the risk of islet autoimmun-

ity. In childhood (1-6 years of age), CLA showed an inverse association with islet autoimmunity (Table 3). In 


\begin{tabular}{|c|c|c|}
\hline & $\begin{array}{l}\text { Case children } \\
\text { Total } n=398\end{array}$ & $\begin{array}{l}\text { Control children } \\
\text { Total } n=1178\end{array}$ \\
\hline \multicolumn{3}{|l|}{ Clinical center, $\mathbf{n}(\%)$} \\
\hline Colorado & $56(14.1)$ & $162(13.8)$ \\
\hline Georgia & $27(6.8)$ & $78(6.6)$ \\
\hline Washington & $36(9.1)$ & $107(9.1)$ \\
\hline Finland & $113(28.4)$ & $339(28.8)$ \\
\hline Germany & $35(8.8)$ & $105(8.9)$ \\
\hline Sweden & $131(32.9)$ & $387(32.9)$ \\
\hline \multicolumn{3}{|l|}{ Sex, n (\%) } \\
\hline Female & $178(44.7)$ & $530(45.0)$ \\
\hline Male & $220(55.3)$ & $648(55.0)$ \\
\hline \multicolumn{3}{|l|}{ Status regarding first degree relative } \\
\hline First degree relative with type 1 diabetes & $88(22.1)$ & $259(22.0)$ \\
\hline General population & $310(77.9)$ & $917(78.0)$ \\
\hline \multicolumn{3}{|l|}{ HLA genotype, n (\%) } \\
\hline High risk (DR3/4) & $210(52.8)$ & $420(35.7)$ \\
\hline Moderate risk (other genotypes) & $187(47.0)$ & $747(63.4)$ \\
\hline Missing & $1(0.2)$ & $11(0.9)$ \\
\hline \multicolumn{3}{|l|}{ Ancestry, mean (SD) } \\
\hline Principal component 1 & $0.0017(0.0074)$ & $0.0013(0.0078)$ \\
\hline Principal component 2 & $-0.0003(0.0109)$ & $-0.0016(0.0094)$ \\
\hline \multicolumn{3}{|l|}{ Breastfed, n (\%) } \\
\hline At 3 months & $307(77.1)$ & $903(76.7)$ \\
\hline At 6 months & $252(63.3)$ & $778(66.0)$ \\
\hline Missing information & $1(0.3)$ & $4(0.3)$ \\
\hline \multicolumn{3}{|l|}{ Weight z score, mean (SD) } \\
\hline At 3 months & $0.68(0.95)$ & $0.41(1.03)$ \\
\hline At 6 months & $0.47(1.00)$ & $0.24(1.01)$ \\
\hline Over $1-6$ years & $0.20(1.04)$ & $0.01(0.99)$ \\
\hline
\end{tabular}

Table 1. Characteristics of TEDDY children with islet autoimmunity and control children.

contrast, higher stearic (18:0) and nervonic (24:1n-9) acids and a high ratio of $n-6: n-3$ PUFA were associated with an increased risk of islet autoimmunity. Furthermore, stearic acid (18:0), cis vaccenic acid (18:1n-7), and dimethylacetal form of 18:0 (DMA18) were associated with a higher risk of multiple islet autoimmunity in childhood (Supplementary information Table 2). A high ratio of $n-6: n-3$ PUFA was associated with an increased risk of IAA first (Supplementary information Table 3), while there were no associations for GADA first (Supplementary information Table 4).

None of the false discovery rate adjusted $p$ values for the associations between fatty acids and the risk of islet autoimmunity, multiple islet autoimmunity, IAA first or GADA first were statistically significant.

\section{Discussion}

Our study showed some associations between erythrocyte fatty acid composition and the risk of islet autoimmunity. EPA $(20: 5 n-3)$ and DPA $(22: 5 n-3)$ in early infancy were associated with a lower risk of islet autoimmunity. These fatty acids originate from the diet, but are synthesized endogenously from ALA $(18: 3 n-3)$ also. ALA itself, as well as LA $(18: 2 n-6)$ in early infancy, showed protective associations with islet autoimmunity in non-breastfed infants. The even-chain SFAs palmitic (16:0) and stearic acid (18:0) and MUFAs oleic (18:1n-9) and nervonic acid $(24: 1 n-9)$ in infancy or childhood, reflecting mostly endogenous biosynthesis in the liver, were associated with an increased risk. On the other hand, CLA $(18: 2 n-7)$ in childhood, obtained from dairy or synthesized endogenously, was associated with a lower risk. The observed associations were, however, not consistent across age. Furthermore, associations between fatty acids and islet autoimmunity differed by the type of outcome (islet autoimmunity, multiple, IAA first, and GADA first).

The results support the view that long-chain $n-3$ PUFAs are protective, especially at an early age. They may affect the activation and development of the immune system in infancy, the maturation of the gut such as microbiota, permeability, and barrier function as well as inflammatory responses, with long-term consequences ${ }^{1}$. Our results are in line with some animal studies ${ }^{28}$ as well as two prospective studies ${ }^{5-7}$ although different $n-3$ fatty acids (ALA, EPA, DPA, DHA) were associated with reduced risk in the different studies. This may be explained by differences in exposure measurements, outcomes and supplementation policies. The fact that our study indicates a protective role for EPA and DPA, and results from the DAISY study for DPA ${ }^{6}$, raises the question whether infants at risk of type 1 diabetes might benefit from supplementation with EPA and DPA also, not 


\begin{tabular}{|c|c|c|c|c|c|c|}
\hline \multirow{3}{*}{$\begin{array}{l}\text { Relative percentage of total fatty acids in } \\
\text { erythrocyte membrane }\end{array}$} & \multicolumn{6}{|c|}{ Islet autoimmunity, cases $n=398$} \\
\hline & \multicolumn{2}{|l|}{3 months } & \multicolumn{2}{|l|}{6 months } & \multicolumn{2}{|c|}{ Mean over 1-6 years } \\
\hline & OR $(95 \% \mathrm{CI})^{\mathrm{a}}$ & $p$ value & OR $(95 \% \mathrm{CI})^{\mathrm{a}}$ & $p$ value & OR $(95 \% \mathrm{CI})^{\mathrm{a}}$ & $p$ value \\
\hline \multicolumn{7}{|l|}{ SFA } \\
\hline Myristic acid 14:0 & $0.95(0.52-1.74)$ & 0.861 & $1.26(0.74-2.13)$ & 0.395 & $0.87(0.42-1.81)$ & 0.706 \\
\hline Pentadecanoic acid 15:0 & $1.19(0.68-2.07)$ & 0.550 & $0.94(0.53-1.67)$ & 0.821 & $0.54(0.27-1.05)$ & 0.068 \\
\hline Palmitic acid 16:0 & $2.31(0.89-5.99)$ & 0.085 & $3.35(1.30-8.65)$ & 0.013 & $3.18(0.82-12.41)$ & 0.096 \\
\hline Heptadecanoic acid 17:0 & $1.95(0.83-4.60)$ & 0.125 & $1.19(0.55-2.58)$ & 0.655 & $0.88(0.31-2.50)$ & 0.808 \\
\hline iso - heptadecanoic acid i17:0 & $0.98(0.76-1.27)$ & 0.882 & $0.87(0.68-1.11)$ & 0.264 & $0.68(0.45-1.02)$ & 0.061 \\
\hline Stearic acid 18:0 & $2.40(0.81-7.16)$ & 0.115 & $2.21(0.71-6.85)$ & 0.170 & $4.70(1.48-14.89)$ & 0.009 \\
\hline Eicosanoid acid 20:0 & $1.34(0.76-2.37)$ & 0.309 & $1.26(0.70-2.26)$ & 0.441 & $1.26(0.59-2.68)$ & 0.546 \\
\hline Docosanoic acid 22:0 $0^{\mathrm{b}}$ & $1.23(0.70-2.16)$ & 0.478 & $1.34(0.72-2.50)$ & 0.348 & $0.99(0.48-2.05)$ & 0.978 \\
\hline Tetracosanic acid 24:0 & $1.46(0.81-2.62)$ & 0.206 & $1.50(0.84-2.67)$ & 0.167 & $1.50(0.74-3.03)$ & 0.261 \\
\hline \multicolumn{7}{|l|}{ MUFA } \\
\hline Palmitoleic acid $16: 1 n-7$ & $1.23(0.77-1.96)$ & 0.395 & $1.03(0.63-1.66)$ & 0.920 & $0.63(0.33-1.20)$ & 0.160 \\
\hline Cis vaccenic acid $18: 1 n-7$ & $1.54(0.62-3.82)$ & 0.355 & $1.84(0.68-4.97)$ & 0.231 & $1.43(0.42-4.86)$ & 0.569 \\
\hline Oleic acid $18: 1 n-9$ & $2.45(1.23-4.88)$ & 0.011 & $1.58(0.75-3.32)$ & 0.229 & $1.59(0.57-4.44)$ & 0.379 \\
\hline $11-$ eicosenoic acid 20:1n-9 & $1.24(0.84-1.84)$ & 0.285 & $1.19(0.78-1.81)$ & 0.413 & $1.61(0.87-2.98)$ & 0.131 \\
\hline Nervonic acid $24: 1 n-9$ & $1.19(0.70-2.02)$ & 0.519 & $1.54(0.91-2.60)$ & 0.104 & $2.04(1.05-3.96)$ & 0.035 \\
\hline \multicolumn{7}{|l|}{ n-6 PUFA } \\
\hline LA $18: 2 n-6^{c}$ & $0.72(0.39-1.36)$ & 0.314 & $1.23(0.64-2.37)$ & 0.527 & $2.32(0.99-5.41)$ & 0.052 \\
\hline DGLA $20: 3 n-6$ & $0.78(0.45-1.37)$ & 0.388 & $0.76(0.43-1.35)$ & 0.350 & $1.18(0.63-2.23)$ & 0.603 \\
\hline AA $20: 4 n-6$ & $0.74(0.38-1.46)$ & 0.388 & $0.79(0.41-1.51)$ & 0.418 & $1.40(0.65-3.00)$ & 0.388 \\
\hline Adrenic acid 22:4n-6 & $1.13(0.60-2.13)$ & 0.701 & $1.32(0.76-2.30)$ & 0.322 & $1.92(0.96-3.87)$ & 0.067 \\
\hline \multicolumn{7}{|l|}{ n-3 PUFA } \\
\hline ALA $18: 3 n-3^{\text {d }}$ & $0.85(0.57-1.28)$ & 0.445 & $0.85(0.57-1.25)$ & 0.399 & $1.14(0.72-1.80)$ & 0.583 \\
\hline EPA 20:5n-3 & $0.67(0.49-0.91)$ & 0.011 & $0.82(0.61-1.10)$ & 0.190 & $0.73(0.51-1.05)$ & 0.090 \\
\hline DPA $22: 5 n-3$ & $0.43(0.25-0.74)$ & 0.002 & $0.66(0.41-1.06)$ & 0.082 & $0.83(0.43-1.62)$ & 0.585 \\
\hline DHA $22: 6 n-3$ & $0.97(0.58-1.65)$ & 0.919 & $0.87(0.52-1.46)$ & 0.604 & $0.99(0.57-1.73)$ & 0.976 \\
\hline \multicolumn{7}{|l|}{ Other } \\
\hline CLA $18: 2 n-7 \mathrm{ct} / \mathrm{tc} 10,12$ & $1.00(0.77-1.30)$ & 0.985 & $0.89(0.70-1.14)$ & 0.369 & $0.66(0.47-0.94)$ & 0.021 \\
\hline DMA16 & $0.98(0.46-2.08)$ & 0.954 & $1.13(0.51-2.53)$ & 0.758 & $1.28(0.50-3.30)$ & 0.612 \\
\hline DMA18 & $0.75(0.32-1.78)$ & 0.518 & $0.94(0.35-2.53)$ & 0.899 & $2.03(0.59-6.98)$ & 0.260 \\
\hline Ratio $n-6: n-3$ PUFA & $1.01(0.91-1.11)$ & 0.908 & $1.09(0.94-1.26)$ & 0.260 & $1.20(1.01-1.41)$ & 0.036 \\
\hline
\end{tabular}

Table 2. The risk of islet autoimmunity associated with erythrocyte fatty acid status in TEDDY nested case-

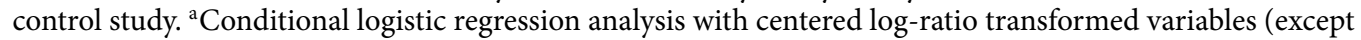
for the ratio of sum $n-6$ and sum $n-3$ ) was adjusted for HLA genotype DR3/4, ancestry (PC1 and PC2), and weight $\mathrm{z}$-score. ${ }^{\mathrm{b}}$ Docosanoid acid (22:0) showed interaction with breastfeeding at 3 months of age $(p=0.027)$. ${ }^{c}$ LA showed interaction with any breastfeeding at 3 months of age $(p=0.038) .{ }^{\mathrm{d}}$ ALA showed interaction with any breastfeeding at 3 months of age $(p=0.024)$.

just DHA. The question is justified because EPA and DPA are precursors of different lipid mediators compared to $\mathrm{DHA}^{29,30}$. Altogether, the results indicate a possibility for preventive interventions by modification of fatty acid composition of early diets (e.g. infant formulas or breastmilk through changes in maternal diet). However, in the TEDDY study maternal intake of $n-3$ fatty acid supplementation during pregnancy was not associated with the risk of islet autoimmunity in the offspring ${ }^{31}$. The ratio of $n-6: n-3$ PUFA showed a positive association with islet autoimmunity and IAA first outcome in children 1-6 years of age suggesting that $n-3$ PUFA may be protective among older children also. Furthermore, the results suggest that $n-3$ PUFA may protect particularly against the development of primary insulin autoimmunity, which is in line with earlier findings ${ }^{7}$.

An important finding in this study was that the major even-chain SFAs [palmitic (16:0), stearic (18:0)], and MUFAs [oleic $(18: 1 n-9)$ and nervonic $(24: 1 n-9)$ acids], were associated with an increased risk of islet autoimmunity. Furthermore, for the multiple islet autoimmunity endpoint, stearic (18:0) and cis vaccenic acid $(18: 1 n-7)$ showed increased risk. The above-mentioned fatty acids are mainly produced endogenously in the liver from shorter-chain fatty acids, as well as by de novo lipogenesis ${ }^{32}$. The increase in SFA and MUFA levels may reflect changes taking place in fatty acid metabolism, before islet autoimmunity. Interestingly, similar associations have been observed for type 2 diabetes in large prospective cohorts ${ }^{32,33}$ possibly reflecting some of the pathogenic disturbances caused by a failure in insulin secretion and signaling ${ }^{24}$. Even-chain SFAs could also have detrimental effects per se, e.g. palmitic acid (16:0) has been associated with activation of inflammatory cytokines and lipotoxicity in pancreatic beta cells ${ }^{34}$. 


\begin{tabular}{|c|c|c|c|c|}
\hline & \multicolumn{2}{|l|}{ At 3 months } & \multicolumn{2}{|l|}{ At 6 months } \\
\hline & Parameter estimate (SE) & $p$ value $^{\mathrm{a}}$ & Parameter estimate (SE) & $p$ value $^{\mathrm{a}}$ \\
\hline \multicolumn{5}{|l|}{ SFA } \\
\hline Myristic acid 14:0 & $-0.11(0.02)$ & $<0.0001$ & $0.03(0.02)$ & 0.090 \\
\hline Pentadecanoic acid 15:0 & $0.21(0.02)$ & $<0.0001$ & $0.22(0.02)$ & $<0.0001$ \\
\hline Palmitic acid 16:0 & $-0.25(0.01)$ & $<0.0001$ & $-0.19(0.01)$ & $<0.0001$ \\
\hline Heptadecanoic acid 17:0 & $0.09(0.02)$ & $<0.0001$ & $0.14(0.01)$ & $<0.0001$ \\
\hline iso-heptadecanoic acid il7:0 & $0.91(0.03)$ & $<0.0001$ & $0.78(0.03)$ & $<0.0001$ \\
\hline Stearic acid 18:0 & $-0.12(0.01)$ & $<0.0001$ & $-0.09(0.01)$ & $<0.0001$ \\
\hline Eicosanoid acid 20:0 & $-0.29(0.02)$ & $<0.0001$ & $-0.24(0.02)$ & $<0.0001$ \\
\hline Docosanoic acid 22:0 & $-0.18(0.02)$ & $<0.0001$ & $-0.14(0.02)$ & $<0.0001$ \\
\hline Tetracosanic acid 24:0 & $-0.22(0.02)$ & $<0.0001$ & $-0.18(0.02)$ & $<0.0001$ \\
\hline \multicolumn{5}{|l|}{ MUFA } \\
\hline Palmitoleic acid $16: 1 n-7$ & $0.34(0.03)$ & $<0.0001$ & $0.22(0.02)$ & $<0.0001$ \\
\hline Cis vaccenic acid $18: 1 n-7$ & $0.10(0.01)$ & $<0.0001$ & $0.06(0.01)$ & $<0.0001$ \\
\hline Oleic acid $18: 1 n-9$ & $-0.25(0.01)$ & $<0.0001$ & $-0.21(0.01)$ & $<0.0001$ \\
\hline $11-$ eicosenoic acid $20: 1 n-9$ & $-0.32(0.03)$ & $<0.0001$ & $-0.32(0.02)$ & $<0.0001$ \\
\hline Nervonic acid 24:1n-9 & $-0.34(0.02)$ & $<0.0001$ & $-0.28(0.02)$ & $<0.0001$ \\
\hline \multicolumn{5}{|l|}{$\mathrm{n}-6$ PUFA } \\
\hline LA $18: 2 n-6$ & $-0.34(0.02)$ & $<0.0001$ & $-0.28(0.01)$ & $<0.0001$ \\
\hline DGLA $20: 3 n-6$ & $-0.04(0.02)$ & 0.042 & $-0.09(0.02)$ & $<0.0001$ \\
\hline AA $20: 4 n-6$ & $-0.08(0.02)$ & $<0.0001$ & $-0.11(0.01)$ & $<0.0001$ \\
\hline Adrenic acid $22: 4 n-6$ & $-0.26(0.02)$ & $<0.0001$ & $-0.29(0.02)$ & $<0.0001$ \\
\hline \multicolumn{5}{|l|}{ n-3 PUFA } \\
\hline ALA $18: 3 n-3$ & $-0.37(0.03)$ & $<0.0001$ & $-0.34(0.02)$ & $<0.0001$ \\
\hline EPA 20:5n-3 & $0.57(0.05)$ & $<0.0001$ & $0.48(0.04)$ & $<0.0001$ \\
\hline DPA $22: 5 n-3$ & $0.31(0.02)$ & $<0.0001$ & $0.32(0.02)$ & $<0.0001$ \\
\hline DHA $22: 6 n-3$ & $0.03(0.02)$ & 0.118 & $-0.01(0.02)$ & 0.679 \\
\hline \multicolumn{5}{|l|}{ Other } \\
\hline CLA $18: 2 n-7 \mathrm{ct} / \mathrm{tc} 10,12$ & $0.82(0.04)$ & $<0.0001$ & $0.73(0.03)$ & $<0.0001$ \\
\hline DMA16 & $-0.16(0.01)$ & $<0.0001$ & $-0.16(0.01)$ & $<0.0001$ \\
\hline DMA18 & $-0.06(0.01)$ & $<0.0001$ & $-0.06(0.01)$ & $<0.0001$ \\
\hline Ratio $n-6: n-3$ PUFA & $-1.36(0.12)$ & $<0.0001$ & $-1.09(0.07)$ & $<0.0001$ \\
\hline
\end{tabular}

Table 3. The difference between fatty acid status of breastfed and not breastfed children at the age of 3 and

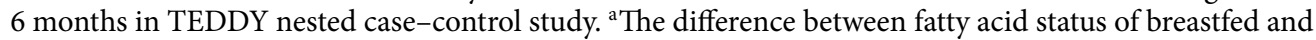
not breastfed children at the age of 3 and 6 month was tested by fitting a linear regression model for CLR transformed fatty acid (except for the ratio of sum $n-6$ and sum $n-3$ ), adjusted for case-control status.

Breastfeeding status affected erythrocyte fatty acid composition in infants in the current study, which is in line with previous findings for serum fatty acids ${ }^{7}$. This is probably explained by differences in fatty acid content of breast milk and infant formula, but may also be caused by some other differences between the breastfed and formula-fed infants. Interestingly, breastfeeding in early infancy modified the association between ALA and LA status and the risk of islet autoimmunity. Higher ALA and LA status showed an inverse association in non-breastfed infants, while no association was seen in breastfed infants. The results indicate that an adequate intake of these essential fatty acids is even more important for infants not receiving any breast milk, and emphasize importance of fatty acid composition of infant formulas, the main source of the essential fatty acids in non-breastfed infants.

In our study, CLA $(18: 2 n-7)$ was associated with a lower risk of islet autoimmunity in children aged 1-6 years. The main dietary source of CLA is dairy products, although it is also derived from fish and meat and it is produced endogenously to some degree ${ }^{35}$. CLA has been shown to exhibit various anti-inflammatory ${ }^{36}$, antiobesogenic and type 2 antidiabetic properties ${ }^{37}$. However, the protective association observed in our study may also be a consequence of increased $n-3$ PUFA levels. CLA supplementation has been shown to increase plasma levels of EPA, for instance ${ }^{38,39}$. Our finding does not support the earlier prospective observation of positive associations between serum CLA and some dairy biomarkers and the risk of advanced islet autoimmunity ${ }^{9}$.

Strengths of the study include a nested case-control design within a large-scale birth cohort, a high number of islet autoimmunity cases, as well as prospectively collected data. Furthermore, we used fatty acid biomarkers, which reflect long-term dietary intake, biosynthesis, and metabolism. In addition, we analyzed a relatively large number of medium to long chain-length fatty acids from several biosynthetic pathways. We adjusted the results with weight because it is associated with both type 1 diabetes development ${ }^{40,41}$ and status of some of the fatty acids. The effect of weight adjustment was, however, relatively small. It can be considered a limitation that our 
421047 newborns from general population and a first degree relative with type 1 diabetes were screened for HLA

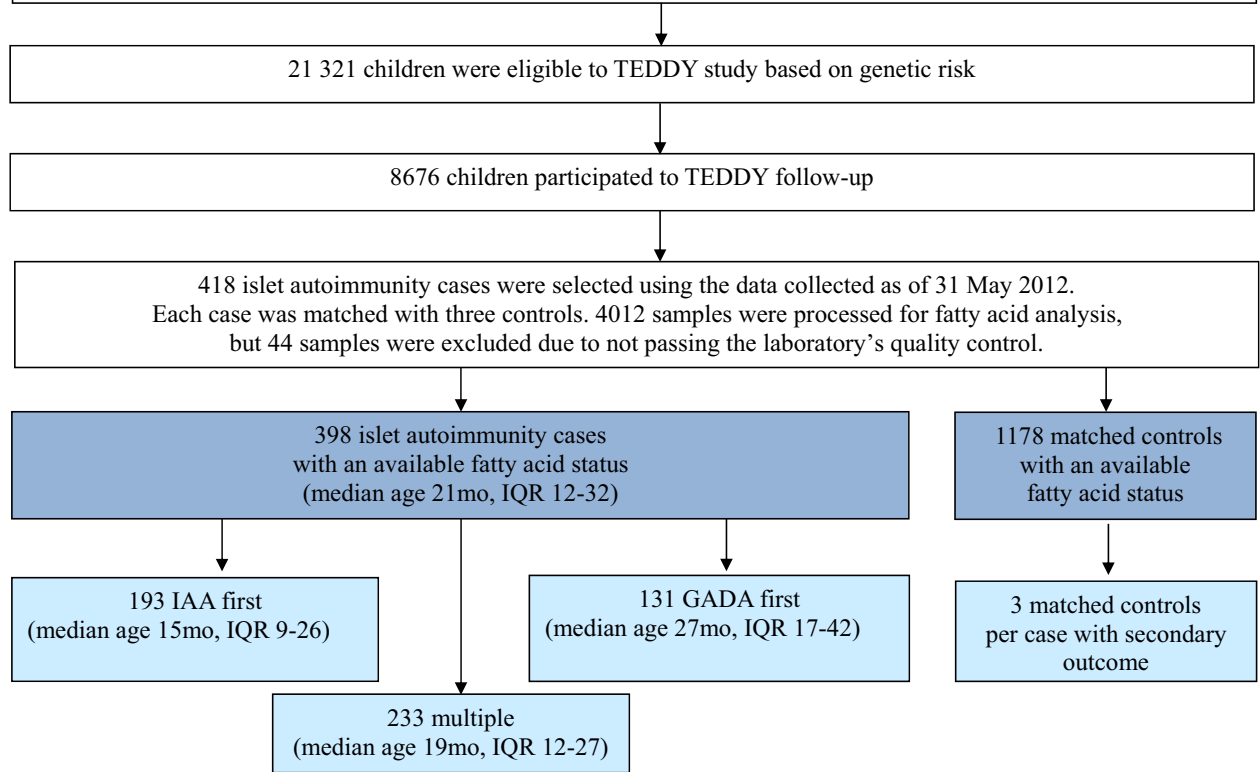

Figure 1. Flow chart of the study participants.

study design does not allow us to draw causal inferences about the observed associations between erythrocyte fatty acid levels and the risk of islet autoimmunity. Further, we did not analyze maternal or child dietary intake of fatty acids. However, this will be done in future research. Also, our study population was selected on the basis of HLA-conferred risk of type 1 diabetes, which limits its generalizability to the whole population.

The current results confirm earlier prospective findings that long-chain $n-3$ PUFA may protect from islet autoimmunity indicating possibility for early dietary intervention in terms of prevention. In addition, changes in the metabolism or intake of other fatty acids, such as even-chain SFAs and MUFAs, and CLA, may precede islet autoimmunity. Further studies are warranted to elucidate the role of individual fatty acids and fatty acid metabolism in type 1 diabetes etiology.

\section{Methods}

TEDDY cohort. The current study was carried out in a nested case-control design within the international prospective the Environmental Determinants of Diabetes in the Young (TEDDY) birth cohort of children with increased genetic risk for type 1 diabetes. The study population was recruited between September 2004 and February 2010 in six clinical sites from the U.S. (Colorado, Georgia and Washington), Finland, Sweden, and Germany. The criteria for increased genetic risk were defined by HLA-associated risk genotypes separately for children from general population and children having a first degree relative with type 1 diabetes. In the general population, HLA-associated risk genotypes were DR3/4, DR3/3, DR4/4 and DR4/8 ${ }^{42}$. Additional eligible genotypes were DR4/1, DR4/13, DR4/9, and DR3/9 in infants with first degree relative with type 1 diabetes. Of the screened 421047 newborns, 21321 were eligible based on the genetic risk and, of them 8676 participated to the follow up before age of 4 months (Fig. 1). Children are followed until the age of 15 years or type 1 diabetes diagnose at 3-6 months intervals. Autoantibodies for insulin autoantibodies (IAA), glutamate decarboxylase (GADA) and islet antigen 2 (IA-2A) were measured. Islet autoimmunity was defined as being persistent confirmed positive for at least one autoantibody out of the three measured. Written informed consent was obtained for all children from a parent and/or legal guardian. All methods were carried out in accordance with relevant guidelines and regulations. The TEDDY study was approved by the following ethical institutional review boards: the Colorado Multiple Institutional Review Board, the Hospital District of Southwest Finland Committee on Ethics, the University of Florida Health Center Institutional Review Board, the Augusta University Institutional Review Board (Georgia), the Ethik-Kommission der Bayerischen Landesarztekammer (Germany), the University of Pittsburgh Institutional Review Board, the Lund University Committee for Continuing Ethical Review (Sweden), the Western Institutional Review Board (Washington), and the University of South Florida Institutional Review Board. The study is also monitored by an External Evaluation Committee formed by the U.S. National Institutes of Health.

A nested case-control design and outcomes. Children's erythrocytes' fatty acid composition was analyzed in a nested case-control design as described previously ${ }^{43}$. Matching factors were the clinical site, sex and family history of type 1 diabetes (first degree relative vs. not). A control was defined as a participant who had not developed persistent islet autoimmunity by the time when the corresponding matched case developed it, within \pm 45 days of the event time. The nested case-control set was based on the data collected as of 31 May 2012 . 
The study included 398 persistent islet autoimmunity cases with an available fatty acid status (385 cases with three controls; 10 cases with two controls; 3 cases with one control) (Fig. 1). In islet autoimmunity cases, median age of seroconversion was 21 months (interquartile range 12-32 months). Multiple islet autoimmunity (repeated positivity for at least two autoantibodies), primary positivity for IAA alone (IAA first), and GADA alone (GADA first) were analyzed as secondary outcomes. From 398 islet autoimmunity cases 233 had multiple islet autoimmunity, 193 had IAA first and 131 had GADA first outcomes. For multiple islet autoimmunity median age was 19 months (interquartile range 12-27), for IAA first 15 months (9-26 months) and for GADA first 27 months (17-42 months).

Erythrocyte sample collection, processing and measurement of fatty acids. Blood samples were obtained from the children by venipuncture at the age of 3 and 6 months and $1,2,3,4,5$, and 6 years at clinic visits. For the participants living far away from their nearest TEDDY clinic, a family periatrician collected the blood samples, which were sent to the TEDDY clinic within $24 \mathrm{~h}$ for processing (long distance protocol). All samples were aliquoted into dedicated, barcoded, and color-coded cryovials. To the blood sample used for fatty acid analysis, 2-propanol with $50 \mathrm{mg} / \mathrm{L}$ of butylated hydroxytoluene were added. The samples were then shipped frozen to the TEDDY Repository and immediately stored at $-80^{\circ} \mathrm{C}$. Collection and processing of samples are previously described in more detail ${ }^{44}$.

Fatty acids were analysed from erythrocytes by a gas chromatographic method modified from previously published methods ${ }^{45,46}$. Erythrocyte fatty acid composition was analysed using an Agilent 6890 gas chromatograph (Hewlett Packard, Palo Alto, CA, USA) with a split injector and hydrogen as the carrier gas. We employed a capillary column Omegawax 320 (length: $30 \mathrm{~m}$, I.D.: $0.32 \mathrm{~mm}$, phase layer: $0.25 \mu \mathrm{m}$; Supelco, Bellefonte, PA, USA). The percentage composition of fatty acid methyl esters was normalized to $100 \%$ in each sample. Samples of the cases and their controls at each age point were processed in the same batch to minimize potential batch effects. The laboratory was blinded regarding the case-control status of the samples. Total 4012 samples were processed for the islet autoimmunity analysis, but 44 samples were excluded due to not passing the laboratory's quality control. The median number of analyzed samples per child was $3(\min =1, \max =7)$. We determined altogether 25 different fatty acids.

Dietary data. We collected information about breastfeeding duration, which was asked at the 3 and 6 months clinic visits. Parents or primary caretaker recorded the infant feeding information in a notebook that was given at the first clinical visit at 3 months. Clinical staff checked the booklet together with the primary caretaker at every clinical visit and entered the dietary information into the TEDDY database. The definition of any breastfeeding included breastfeeding, even in small amounts, and in combination with other foods. In the statistical analyses we used two categories for any breastfeeding: breastfed/not breastfed at cross-sectional time point either 3 or 6 months of age.

Genetic measurements. Children in the study cohort were genotyped for the major type 1 diabetes associated class II haplotypes as well as for single-nucleotide polymorphisms (SNPs) defining type 1 diabetes risk outside HLA region ${ }^{47}$. Ancestry was estimated based on the principal components analysis (PCA $)^{48}$ from the ImmunoChip data using the entire cohort. EIGENSTRAT software was used after selecting one subject per family. Two largest principal components were used in this study for defining population stratification.

Statistical analysis. Fatty acid status for each child was generated as a percentage of the total 25 fatty acids. Since the sum is restricted to 100 , the fatty acid status carries only a relative information, which may produce spurious findings without data normalization. Thus, we used the centered log-ratio (CLR) transformed fatty acid status for statistical comparisons, except for the ratio of sum $n-6$ and sum $n-3$ PUFA. Sum of $n-6$ PUFA was obtained by summing up LA, dihomogammalinolenic acid (DGLA), arachidonic acid (AA) and adrenic acid. Sum of $n-3$ PUFA was the sum of ALA, EPA, DPA and DHA. As the change after 1 year old was ignorable, we analyzed fatty acid status at early age ( 3 and 6 months), along with the average status from 1 to 6 years old. Conditional logistic regression examined the association between islet autoimmunity and fatty acid status after adjusting for HLA genotype, ancestry and weight $\mathrm{z}$-score at the age corresponding to fatty acid status. The average weight from 1 to 6 years old was adjusted for the average status from 1 to 6 years old. Weight $\mathrm{z}$ score was obtained from Centers for Disease Control and Prevention standardized growth charts. Interaction between fatty acid status at early age and whether any breastfeeding took place at the corresponding age on the risk of islet autoimmunity was examined by testing an interaction term in the conditional logistic regression model. One unit change in a CLR transformed fatty acid status corresponds to the fatty acid status in percentage times 1.83. Association between fatty acid status at early age and the corresponding breastfeeding status was assessed using a linear regression model adjusted for the case-control status. Two-sided $p$ values are reported. Statistical significance was determined when the $p$ value was $<0.05$. All statistical analyses were performed using SAS version 9.4 (SAS Institute Inc., Cary, NC).

Since 26 defined fatty acids were analyzed for each outcome, false discovery rate adjusted $p$ values were calculated for multiple testing correction ${ }^{49}$.

\section{Data availability}

The datasets generated and analyzed during the current study will be made available in the NIDDK Central Repository at https://www.niddkrepository.org/studies/teddy. 
Received: 2 July 2020; Accepted: 11 January 2021

Published online: 11 February 2021

\section{References}

1. Radzikowska, U. et al. The influence of dietary fatty acids on immune responses. Nutrients 11, 2990. https://doi.org/10.3390/nu111 22990 (2019).

2. Innis, S. M. Metabolic programming of long-term outcomes due to fatty acid nutrition in early life. Matern. Child. Nutr. 7(Suppl 2), 112-123 (2011).

3. Calder, P. C. Feeding the immune system. Proc. Nutr. Soc. 72, 299-309 (2013).

4. Calder, P. C. N-3 fatty acids, inflammation and immunity: new mechanisms to explain old actions. Proc. Nutr. Soc. 72, 326-336 (2013).

5. Norris, J. M. et al. Omega-3 polyunsaturated fatty acid intake and islet autoimmunity in children at increased risk for type 1 diabetes. JAMA 298, 1420-1428 (2007).

6. Norris, J. M. et al. Erythrocyte membrane docosapentaenoic acid levels are associated with islet autoimmunity: the Diabetes Autoimmunity Study in the Young. Diabetologia 57, 295-304 (2014).

7. Niinisto, S. et al. Fatty acid status in infancy is associated with the risk of type 1 diabetes-associated autoimmunity. Diabetologia 60, 1223-1233 (2017).

8. Miller, M. R. et al. Erythrocyte membrane omega-3 fatty acid levels and omega-3 fatty acid intake are not associated with conversion to type 1 diabetes in children with islet autoimmunity: the Diabetes Autoimmunity Study in the Young (DAISY). Pediatr. Diabetes 12, 669-675 (2011).

9. Virtanen, S. M. et al. Serum fatty acids and risk of advanced beta-cell autoimmunity: a nested case-control study among children with HLA-conferred susceptibility to type I diabetes. Eur. J. Clin. Nutr. 64, 792-799 (2010).

10. Oresic, M. et al. Cord serum lipidome in prediction of islet autoimmunity and type 1 diabetes. Diabetes 62, 3268-3274 (2013).

11. Pflueger, M. et al. Age- and islet autoimmunity-associated differences in amino acid and lipid metabolites in children at risk for type 1 diabetes. Diabetes 60, 2740-2747 (2011).

12. La Torre, D. et al. Decreased cord-blood phospholipids in young age-at-onset type 1 diabetes. Diabetes 62, 3951-3956 (2013).

13. Oresic, M. et al. Dysregulation of lipid and amino acid metabolism precedes islet autoimmunity in children who later progress to type 1 diabetes. J. Exp. Med. 205, 2975-2984 (2008).

14. Johnson, R. K. et al. Metabolite-related dietary patterns and the development of islet autoimmunity. Sci. Rep. 9, 14819-14824 (2019).

15. Brenna, J. T., Plourde, M., Stark, K. D., Jones, P. J. \& Lin, Y. H. Best practices for the design, laboratory analysis, and reporting of trials involving fatty acids. Am. J. Clin. Nutr. 108, 211-227 (2018).

16. Greupner, T. et al. Effects of a low and a high dietary LA/ALA ratio on long-chain PUFA concentrations in red blood cells. Food Funct. 9, 4742-4754 (2018).

17. Tu, W. C., Cook-Johnson, R. J., James, M. J., Muhlhausler, B. S. \& Gibson, R. A. Omega-3 long chain fatty acid synthesis is regulated more by substrate levels than gene expression. Prostaglandins Leukot. Essent. Fatty Acids 83, 61-68 (2010).

18. Lankinen, M., Uusitupa, M. \& Schwab, U. Genes and dietary fatty acids in regulation of fatty acid composition of plasma and erythrocyte membranes. Nutrients 10, 1785. https://doi.org/10.3390/nu10111785 (2018).

19. Wolk, A., Furuheim, M. \& Vessby, B. Fatty acid composition of adipose tissue and serum lipids are valid biological markers of dairy fat intake in men. J. Nutr. 131, 828-833 (2001).

20. Pranger, I. G. et al. Fatty acids as biomarkers of total dairy and dairy fat intakes: a systematic review and meta-analysis. Nutr. Rev. 77, 46-63 (2019).

21. Jenkins, B., West, J. A. \& Koulman, A. A review of odd-chain fatty acid metabolism and the role of pentadecanoic Acid (c15:0) and heptadecanoic Acid (c17:0) in health and disease. Molecules 20, 2425-2444 (2015).

22. Lankinen, M. \& Schwab, U. Biomarkers of dairy fat. Am. J. Clin. Nutr. 101, 1101-1102 (2015).

23. Ratnayake, W. M. Concerns about the use of 15:0, 17:0, and trans-16:1n-7 as biomarkers of dairy fat intake in recent observational studies that suggest beneficial effects of dairy food on incidence of diabetes and stroke. Am. J. Clin. Nutr. 101, 1102-1103 (2015).

24. Sanders, F. W. \& De Griffin, J. L. novo lipogenesis in the liver in health and disease: more than just a shunting yard for glucose. Biol. Rev. Camb. Philos. Soc. 91, 452-468 (2016).

25. Leete, P. et al. Studies of insulin and proinsulin in pancreas and serum support the existence of aetiopathological endotypes of type 1 diabetes associated with age at diagnosis. Diabetologia 63, 1258-1267 (2020).

26. Ilonen, J. et al. Patterns of beta-cell autoantibody appearance and genetic associations during the first years of life. Diabetes 62 , 3636-3640 (2013).

27. Krischer, J. P. et al. Genetic and environmental interactions modify the risk of diabetes-related autoimmunity by 6 years of age: the TEDDY Study. Diabetes Care 40, 1194-1202 (2017).

28. $\mathrm{Bi}, \mathrm{X}$. et al. Omega-3 polyunsaturated fatty acids ameliorate type 1 diabetes and autoimmunity. J. Clin. Investig. 127, 1757-1771 (2017).

29. Galli, C. \& Calder, P. C. Effects of fat and fatty acid intake on inflammatory and immune responses: a critical review. Ann. Nutr. Metab. 55, 123-139 (2009).

30. Drouin, G., Rioux, V. \& Legrand, P. The n-3 docosapentaenoic acid (DPA): A new player in the n-3 long chain polyunsaturated fatty acid family. Biochimie 159, 36-48 (2019).

31. Silvis, K. et al. Maternal dietary supplement use and development of islet autoimmunity in the offspring: TEDDY study. Pediatr. Diabetes 20, 86-92 (2019).

32. Ma, W. et al. Prospective association of fatty acids in the de novo lipogenesis pathway with risk of type 2 diabetes: the Cardiovascular Health Study. Am. J. Clin. Nutr. 101, 153-163 (2015).

33. Forouhi, N. G. et al. Differences in the prospective association between individual plasma phospholipid saturated fatty acids and incident type 2 diabetes: the EPIC-InterAct case-cohort study. Lancet Diabetes Endocrinol. 2, 810-818 (2014).

34. Maedler, K. et al. Distinct effects of saturated and monounsaturated fatty acids on beta-cell turnover and function. Diabetes $\mathbf{5 0}$, 69-76 (2001).

35. Wahle, K. W., Heys, S. D. \& Rotondo, D. Conjugated linoleic acids: are they beneficial or detrimental to health?. Prog. Lipid Res. 43, 553-587 (2004).

36. Viladomiu, M., Hontecillas, R. \& Bassaganya-Riera, J. Modulation of inflammation and immunity by dietary conjugated linoleic acid. Eur. J. Pharmacol. 785, 87-95 (2016).

37. Koba, K. \& Yanagita, T. Health benefits of conjugated linoleic acid (CLA). Obes. Res. Clin. Pract. 8, 525 (2014).

38. Attar-Bashi, N. M., Weisinger, R. S., Begg, D. P., Li, D. \& Sinclair, A. J. Failure of conjugated linoleic acid supplementation to enhance biosynthesis of docosahexaenoic acid from alpha-linolenic acid in healthy human volunteers. Prostaglandins Leukot. Essent. Fatty Acids 76, 121-130 (2007).

39. Murru, E. et al. Dietary conjugated linoleic acid-enriched cheeses influence the levels of circulating n-3 highly unsaturated fatty acids in humans. Int. J. Mol. Sci. 19, 1730. https://doi.org/10.3390/ijms19061730 (2018).

40. Hypponen, E. et al. Obesity, increased linear growth, and risk of type 1 diabetes in children. Diabetes Care 23, 1755-1760 (2000). 
41. Elding Larsson, H. et al. Growth and risk for islet autoimmunity and progression to type 1 diabetes in early childhood: the Environmental Determinants of Diabetes in the Young Study. Diabetes 65, 1988-1995 (2016).

42. Hagopian, W. A. et al. The Environmental Determinants of Diabetes in the Young (TEDDY): genetic criteria and international diabetes risk screening of 421000 infants. Pediatr. Diabetes 12, 733-743 (2011).

43. Lee, H. S. et al. Biomarker discovery study design for type 1 diabetes in The Environmental Determinants of Diabetes in the Young (TEDDY) study. Diabetes Metab. Res. Rev. 30, 424-434 (2014).

44. Vehik, K. et al. Methods, quality control and specimen management in an international multicentre investigation of type 1 diabetes: TEDDY. Diabetes Metab. Res. Rev. 29, 557-567 (2013).

45. Rose, H. G. \& Oklander, M. Improved procedure for the extraction of lipids from human erythrocytes. J. Lipid Res. 6, 428-431 (1965).

46. Elorinne, A.-L. et al. Food and nutrient intake and nutritional status of Finnish vegans and non-vegetarians. PLoS ONE 11, $\mathrm{e} 0148235$ (2016).

47. Sharma, A. et al. Identification of non-HLA genes associated with development of islet autoimmunity and type 1 diabetes in the prospective TEDDY cohort. J. Autoimmun. 89, 90-100 (2018).

48. Price, A. L. et al. Principal components analysis corrects for stratification in genome-wide association studies. Nat. Genet. 38, 904-909 (2006).

49. Benjamini, Y. \& Hochberg, Y. Controlling the false discovery rate: a practical and powerful approach to multiple testing. J. R. Stat. Soc. 57, 289-300 (1995).

\section{Acknowledgements}

We express our gratitude to the TEDDY study families for their continued participation in the study. The TEDDY Study Group is acknowledged for collaboration. For biochemical analysis of fatty acids, we acknowledge the Dietary Biomarkers Laboratory: Iris Erlund, Ph.D., Irma Salminen, Jouko Sundvall, Nina Kangas, Petra Arohonka. Finnish Institute for Health and Welfare, Helsinki, Finland.

\section{Author contributions}

S.N. contributed to the study design, analysis, interpretation of data, the drafting of the manuscript, and critical revision of the manuscript. I.E. contributed to the study design, analysis, interpretation of data, and critical revision of the manuscript, and supervised fatty acid laboratory analyses. H.-S.L. performed statistical analysis, contributed to the interpretation of data, and revision of the manuscript. U.U., I.S., C.A.A., H.P., X.L., S.H., J.T., J.X.S., Å.L., A.G.Z., M.R., B.A., J.K., D.G., S.D., N.S., S.R., and W.H. contributed to the acquisition and interpretation of the data and critically reviewed the manuscript. J.M.N. and S.M.V. contributed to the study concept and design, analysis, acquisition and interpretation of data, and critical revision of the manuscript. All authors approved the final version of the article. S.N., H.-S.L., and S.M.V. are the guarantors of this work, had full access to all the data in the study, and take responsibility for the integrity of the data and the accuracy of the data analysis.

\section{Funding}

The TEDDY Study is funded by U01 DK63829, U01 DK63861, U01 DK63821, U01 DK63865, U01 DK63863, U01 DK63836, U01 DK63790, UC4 DK63829, UC4 DK63861, UC4 DK63821, UC4 DK63865, UC4 DK63863, UC4 DK63836, UC4 DK95300, UC4 DK100238, UC4 DK106955, UC4 DK112243, UC4 DK117483, and Contract No. HHSN267200700014C from the National Institute of Diabetes and Digestive and Kidney Diseases (NIDDK), National Institute of Allergy and Infectious Diseases (NIAID), Eunice Kennedy Shriver National Institute of Child Health and Human Development (NICHD), National Institute of Environmental Health Sciences (NIEHS), Centers for Disease Control and Prevention (CDC), and JDRF. This work supported in part by the NIH/NCATS Clinical and Translational Science Awards to the University of Florida (UL1 TR000064) and the University of Colorado (UL1 TR001082) as well as by the Academy of Finland (Grant 276475). Role of the funder/sponsor: The sponsors of this study were represented on the Steering Committee and played a role in the design and conduct of the study; collection, management, analysis, and interpretation of the data; and preparation, review, or approval of the manuscript; and decision to submit the manuscript for publication. The corresponding author had the final decision to submit the manuscript for publication.

\section{Competing interests}

The authors declare no competing interests.

\section{Additional information}

Supplementary Information The online version contains supplementary material available at https://doi. org/10.1038/s41598-021-82200-9.

Correspondence and requests for materials should be addressed to S.N.

Reprints and permissions information is available at www.nature.com/reprints.

Publisher's note Springer Nature remains neutral with regard to jurisdictional claims in published maps and institutional affiliations. 


\begin{abstract}
(c) (i) Open Access This article is licensed under a Creative Commons Attribution 4.0 International License, which permits use, sharing, adaptation, distribution and reproduction in any medium or format, as long as you give appropriate credit to the original author(s) and the source, provide a link to the Creative Commons licence, and indicate if changes were made. The images or other third party material in this article are included in the article's Creative Commons licence, unless indicated otherwise in a credit line to the material. If material is not included in the article's Creative Commons licence and your intended use is not permitted by statutory regulation or exceeds the permitted use, you will need to obtain permission directly from the copyright holder. To view a copy of this licence, visit http://creativecommons.org/licenses/by/4.0/.
\end{abstract}

(C) The Author(s) 2021

\title{
the TEDDY Study Group
}

\section{Colorado Clinical Center}

Aaron Barbour ${ }^{8}$, Kimberly Bautista ${ }^{8}$, Judith Baxter ${ }^{8}$, Daniel Felipe-Morales ${ }^{8}$, Kimberly Driscoll ${ }^{8}$, Brigitte I. Frohnert ${ }^{8}$, Marisa Stahl ${ }^{8}$, Patricia Gesualdo ${ }^{8}$, Michelle Hoffman ${ }^{8}$, Rachel Karban $^{8}$, Edwin Liu ${ }^{8}$, Stesha Peacock ${ }^{8}$, Hanan Shorrosh ${ }^{8}$, Andrea Steck ${ }^{8}$, Megan Stern ${ }^{8}$, Erica Villegas $^{8} \&$ Kathleen Waugh ${ }^{8}$

\section{Finland Clinical Center}

Olli G. Simell ${ }^{6}$, Annika Adamsson ${ }^{15}$, Suvi Ahonen ${ }^{1,13}$, Mari Åkerlund ${ }^{1,13}$, Leena Hakola ${ }^{13}$, Anne Hekkala $^{16,17}$, Henna Holappa ${ }^{16,17}$, Heikki Hyöty ${ }^{13}$, Anni Ikonen ${ }^{16,17}$, Jorma Ilonen ${ }^{6,18}$, Sinikka Jäminki $^{13}$, Sanna Jokipuu ${ }^{15}$, Leena Karlsson ${ }^{15}$, Jukka Kero ${ }^{6,15}$, Miia Kähönen ${ }^{16,17}$, Mikael Knip $^{13}$, Minna-Liisa Koivikko ${ }^{16,17}$, Merja Koskinen ${ }^{13}$, Mirva Koreasalo ${ }^{1,13}$, Kalle Kurppa ${ }^{13}$, Jarita Kytölä ${ }^{13}$, Tiina Latva-Aho ${ }^{16,17}$, Katri Lindfors ${ }^{13}$, Maria Lönnrot ${ }^{13}$, Elina Mäntymäki ${ }^{15}$, Markus Mattila $^{13}$, Maija Miettinen ${ }^{1}$, Katja Multasuo ${ }^{16,17}$, Teija Mykkänen ${ }^{16,17}$, Tiina Niininen ${ }^{13}$, Mia Nyblom $^{13}$, Sami Oikarinen ${ }^{13}$, Paula Ollikainen ${ }^{16}$, Zhian Othmani ${ }^{15}$, Sirpa Pohjola ${ }^{16,17}$, Petra Rajala $^{15}$, Jenna Rautanen ${ }^{1}$, Anne Riikonen ${ }^{1,13}$, Eija Riski ${ }^{15}$, Miia Pekkola ${ }^{13}$, Minna Romo ${ }^{15}$, Satu Ruohonen ${ }^{15}$, Satu Simell ${ }^{6}$, Maija Sjöberg ${ }^{15}$, Aino Stenius ${ }^{16,17}$, Päivi Tossavainen ${ }^{16,17}$, Mari Vähä-Mäkilä6 ${ }^{2}$ Sini Vainionpää ${ }^{15}$, Eeva Varjonen ${ }^{15}$, Riitta Veijola ${ }^{16,17}$ \& Irene Viinikangas ${ }^{16,17}$

${ }^{15}$ Hospital District of Southwest Finland, Turku University Hospital, Turku, Finland. ${ }^{16}$ University of Oulu, Oulu, Finland. ${ }^{17}$ Oulu University Hospital, Oulu, Finland. ${ }^{18}$ University of Kuopio, Kuopio, Finland.

\section{Georgia/Florida Clinical Center}

Desmond Schatz ${ }^{3}$, Diane Hopkins ${ }^{7}$, Leigh Steed ${ }^{7}$, Jennifer Bryant ${ }^{7}$, Katherine Silvis ${ }^{7}$, Michael Haller $^{3}$, Melissa Gardiner ${ }^{7}$, Richard McIndoe ${ }^{7}$, Ashok Sharma ${ }^{7}$, Stephen W. Anderson ${ }^{19}$, Laura Jacobsen $^{3} \&$ John Marks ${ }^{3}$

${ }^{7}$ Medical College of Georgia, Augusta University, Augusta, GA, USA. ${ }^{19}$ Pediatric Endocrine Associates, Atlanta, USA.

\section{Germany Clinical Center}

Ezio Bonifacio ${ }^{20}$, Cigdem Gezginci ${ }^{5}$, Anja Heublein ${ }^{5}$, Eva Hohoff ${ }^{21}$, Annette Knopff ${ }^{5}$, Charlotte Koch $^{5}$, Sibylle Koletzko ${ }^{22}$, Claudia Ramminger ${ }^{5}$, Roswith Roth ${ }^{5}$, Jennifer Schmidt ${ }^{5}$, Marlon Scholz ${ }^{5}$, Joanna Stock ${ }^{5}$, Katharina Warncke ${ }^{5}$, Lorena Wendel ${ }^{5}$ \& Christiane Winkler $^{5}$

${ }^{20}$ Center for Regenerative Therapies, TU Dresden, Dresden, Germany. ${ }^{21}$ Department of Nutritional Epidemiology, University of Bonn, Bonn, Germany. ${ }^{22}$ Dr. Von Hauner Children's Hospital, Department of Gastroenterology, Ludwig Maximillians University Munich, Munich, Germany.

\section{Sweden Clinical Center}

Daniel Agardh", Maria Ask ${ }^{4}$, Rasmus Bennet ${ }^{4}$, Corrado Cilio4, Susanne Dahlberg ${ }^{4}$, Helene Engqvist ${ }^{4}$, Emelie Ericson-Hallström ${ }^{4}$, Annika Björne Fors ${ }^{4}$, Lina Fransson ${ }^{4}$, Thomas Gard $^{4}$, Monika Hansen ${ }^{4}$, Hanna Jisser ${ }^{4}$, Fredrik Johansen ${ }^{4}$, Berglind Jonsdottir ${ }^{4}$, Helena Elding Larsson ${ }^{4}$, Marielle Lindström ${ }^{4}$, Markus Lundgren ${ }^{4}$, Marlena Maziarz ${ }^{4}$, Maria 
Månsson-Martinez ${ }^{4}$, Jessica Melin 4 , Zeliha Mestan ${ }^{4}$, Caroline Nilsson $^{4}$, Karin Ottosson 4 , Kobra Rahmati ${ }^{4}$, Anita Ramelius ${ }^{4}$, Falastin Salami ${ }^{4}$, Anette Sjöberg ${ }^{4}$, Birgitta Sjöberg ${ }^{4}$, Carina Törn ${ }^{4} \&$ Åsa Wimar ${ }^{4}$

Washington Clinical Center

Michael Killian ${ }^{10}$, Claire Cowen Crouch ${ }^{10}$, Jennifer Skidmore ${ }^{10}$, Masumeh Chavoshi ${ }^{10}$, Arlene Meyer ${ }^{10}$, Jocelyn Meyer ${ }^{10}$, Denise Mulenga ${ }^{10}$, Nole Powell ${ }^{10}$, Jared Radtke ${ }^{10}$, Matei Romancik $^{10}$, Shreya Roy ${ }^{10}$, Davey Schmitt ${ }^{10}$ \& Sarah Zink ${ }^{10}$

Pennsylvania Satellite Center

Dorothy Becker ${ }^{23}$, Margaret Franciscus ${ }^{23}$, MaryEllen Dalmagro-Elias Smith ${ }^{23}$, Ashi Daftary ${ }^{23}$, Mary Beth Klein ${ }^{23}$ \& Chrystal Yates ${ }^{23}$

${ }^{23}$ Children's Hospital of Pittsburgh of UPMC, Pittsburgh, USA.

\section{Data Coordinating Center}

Sarah Austin-Gonzalez ${ }^{3}$, Maryouri Avendano ${ }^{3}$, Sandra Baethke ${ }^{3}$, Brant Burkhardt ${ }^{3}$, Martha Butterworth ${ }^{3}$, Joanna Clasen ${ }^{3}$, David Cuthbertson ${ }^{3}$, Christopher Eberhard ${ }^{3}$, Steven Fiske ${ }^{3}$, Jennifer Garmeson ${ }^{3}$, Veena Gowda ${ }^{3}$, Kathleen Heyman ${ }^{3}$, Belinda Hsiao ${ }^{3}$, Christina Karges ${ }^{3}$, Francisco Perez Laras ${ }^{3}$, Qian Li ${ }^{3}$, Shu Liu ${ }^{3}$, Kristian Lynch ${ }^{3}$, Colleen Maguire ${ }^{3}$, Jamie Malloy ${ }^{3}$, Cristina McCarthy ${ }^{3}$, Cassandra Remedios ${ }^{3}$, Chris Shaffer ${ }^{3}$, Laura Smith ${ }^{3}$, Susan Smith ${ }^{3}$, Noah Sulman ${ }^{3}$, Roy Tamura ${ }^{3}$, Dena Tewey ${ }^{3}$, Michael Toth ${ }^{3}$, Kendra Vehik ${ }^{3}$, Ponni Vijayakandipan ${ }^{3}$ \& Jimin Yang ${ }^{3}$

\section{Past staff}

Michael Abbondondolo ${ }^{3}$, Lori Ballard ${ }^{3}$, Rasheedah Brown ${ }^{3}$, Stephen Dankyi ${ }^{3}$, David Hadley ${ }^{3}$, Wendy McLeod ${ }^{3}$, Aubrie Merrell ${ }^{3}$, Steven Meulemans ${ }^{3}$ \& Ryan Quigley ${ }^{3}$

\section{Autoantibody Reference Laboratories}

Liping $\mathrm{Yu}^{8}$, Dongmei Miao ${ }^{8}$, Polly Bingley ${ }^{24}$, Alistair Williams ${ }^{24}$, Kyla Chandler ${ }^{24}$, Ilana Kelland ${ }^{24}$, Yassin Ben Khoud ${ }^{24}$, Huma Zahid ${ }^{24}$ \& Matthew Randell ${ }^{24}$

${ }^{24}$ Bristol Medical School, University of Bristol, Bristol, UK.

Dietary Biomarkers Laboratory

Jouko Sundvall ${ }^{2}$, Nina Kangas ${ }^{2} \&$ Petra Arohonka ${ }^{2}$

HLA Reference Laboratory

Masumeh Chavoshi ${ }^{10}$, Jared Radtke ${ }^{10}$, Sarah Zink ${ }^{10}$, Previously Henry Erlich ${ }^{25}$, Steven J. Mack ${ }^{25}$ \& Anna Lisa Fear ${ }^{25}$

${ }^{25}$ Center for Genetics, Children's Hospital Oakland Research Institute, Oakland, USA.

SNP Laboratory

Wei-Min Chen ${ }^{11}$, Suna Onengut-Gumuscu ${ }^{11}$, Emily Farber ${ }^{11}$, Rebecca Roche Pickin ${ }^{11}$, Jonathan Davis ${ }^{11}$, Jordan Davis ${ }^{11}$, Dan Gallo ${ }^{11}$, Jessica Bonnie ${ }^{11}$ \& Paul Campolieto ${ }^{11}$

Repository

Sandra $\mathrm{Ke}^{26} \&$ Niveen Mulholland ${ }^{26}$

${ }^{26}$ NIDDK Biosample Repository at Fisher BioServices, Rockville, USA. 


\section{Other contributors}

\section{Kasia Bourcier ${ }^{27}$, Thomas Briese ${ }^{28}$, Suzanne Bennett Johnson ${ }^{29}$ \& Eric Triplett $^{30}$}

${ }^{27}$ National Institutes of Allergy and Infectious Diseases, Palo Alto, USA. ${ }^{28}$ Columbia University, New York, USA.

${ }^{29}$ Florida State University, Tallahassee, USA. ${ }^{30}$ University of Florida, Gainesville, USA. 